\title{
Les attentats-suicides, sont-ils irrationnels?
}

\author{
Minh-Hoang Nguyen \\ Centre de Recherche Interdisciplinaire sur la Société \\ Université de Phenikaa \\ Vietnam \\ Hanh-Quyen Tran \\ Chercheuse Indépendente \\ Vietnam \\ 2 septembre, 2021
}

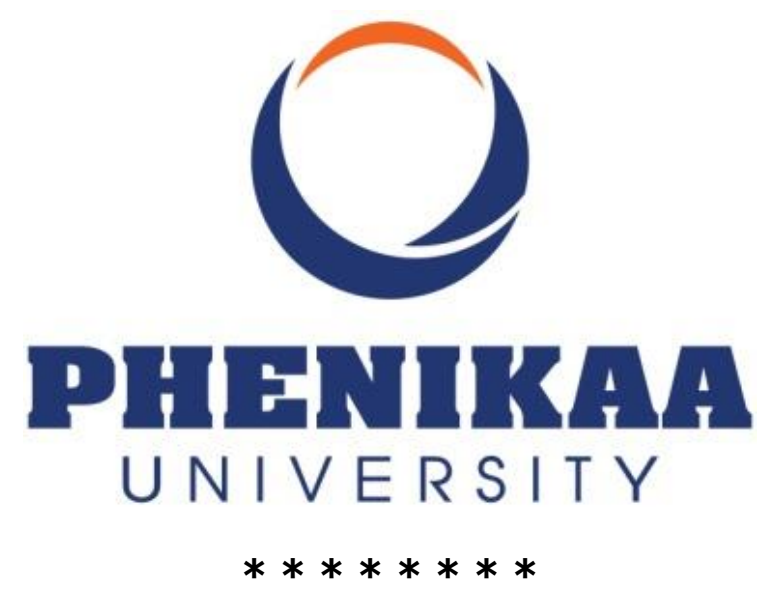

L'armée américaine a rapidement mené une attaque de drone contre un membre de l'État islamique (EI) juste un jour après les attentats suicides à l'aéroport de Kaboul, faisant au moins 175 victimes et 120 blessés [1]. La vengeance des États-Unis pourrait se poursuivre, les attentats-suicides reviendront et un cercle vicieux de la violence continuera [2]. Personne ne sait quand ce cycle prendra fin.

La question est : "Leurs actions sont-elles rationnelles ?"

Du point de vue des États-Unis, leur réaction rapide pour éliminer un membre de l'EI est rationnelle, mais qu'en est-il des anciens attentats-suicides des "terroristes"?

Avec le bon sens, la suicidalité, quelle que soit sa forme (idéation ou comportement), est jugée irrationnelle et généralement condamnée par les autres. Nous pouvons avoir de nombreuses options à choisir dans notre vie, alors pourquoi choisirions-nous de nous suicider? Personne ne choisit de se suicider s'il apprécie sa vie et y trouve un sens. À moins que le coût perçu de la vie ne soit plus important que ses avantages, l'idée suicidaire apparaîtra et sera considérée comme une option [3]. 
De nombreuses choses peuvent avoir un sens dans notre vie, comme la famille, les amis, les loisirs, les religions, les pays, etc. Si toutes ces choses sont déshonorées, ou même détruites, que ferions-nous? Ignorer, se venger ou se suicider? Cela dépend des jugements coûts-avantages de chaque individu. Néanmoins, la négativité de l'esprit humain peut être facilement exploitée par des chefs religieux ayant des intérêts politiques déformés et utilisant le mécanisme de croyance dans le processus psycho-religieux. Les actions radicalisées qui s'ensuivent, comme les attentats suicides, sont parfois inévitables et finissent par renforcer l'effusion de sang [4].

La chose ou l'action que nous considérons comme irrationnelle peut être perçue comme rationnelle par d'autres personnes et simplement parce que les humains ne sont pas toujours du même avis. C'est le cas des États-Unis et des auteurs d'attentats suicides. Peut-être, la communication mutuelle et les activités de renforcement de la confiance sont les clés pour mettre fin à ce cercle vicieux de la violence [4].

\section{Références}

1. Associated Press. (2021). U.S. airstrike targets Islamic State member in Afghanistan. POLITICO. Retrieved from https://www.politico.com/news/2021/08/27/us-airstrike-islamic-stateafghanistan-507062

2. Le TT. (2021). Bloodshed will not stop in a constantly reinforced conflict. OSF Preprints. DOI: $10.31219 /$ osf.io/h96sm

3. Nguyen MH, Le TT, Nguyen HKT, Ho MT, Nguyen HTT, Vuong QH. (2021). Alice in Suicideland: Exploring the suicidal ideation mechanism through the sense of connectedness and help-seeking behaviors. International Journal of Environmental Research and Public Health, 18(7), 3681.

4. Vuong $\mathrm{QH}$, Nguyen $\mathrm{MH}$, Le TT. (2021). A Mindsponge-Based Investigation into the Psycho-Religious Mechanism Behind Suicide Attacks. Warsaw, Poland: De Gruyter. 